\title{
Effect of Manual Acupuncture for Mild-to-Moderate Carpal Tunnel Syndrome: A Systematic Review
}

\author{
Jeong Ho Huh ${ }^{1 \dagger}$, Hye In Jeong ${ }^{2 \dagger}$, Kyeong Han $\mathrm{Kim}^{3,4}$ * \\ ${ }^{1}$ College of Korean Medicine, Woosuk University, Jeonju, Republic of Korea \\ ${ }^{2}$ Department of Preventive Medicine, College of Korean Medicine, Kyung Hee University, Seoul, Republic of Korea \\ ${ }^{3}$ Woosuk Institute of Smart Convergence Life Care (WSCLC), Woosuk University, Wanju, Republic of Korea \\ ${ }^{4}$ Department of Preventive Medicine, College of Korean Medicine, Woosuk University, Wanju, Republic of Korea
}

Received August 30, 2021

Reviewed September 9, 2021

Accepted November 19, 2021

\begin{abstract}
*Corresponding Author
Kyeong Han Kim

Department of Preventive Medicine,

College of Korean Medicine, Woosuk

University, 443 Samnye-ro, Samnye-eup,

Wanju 55338, Republic of Korea

Tel: +82-63-290-9031

E-mail: solip922@hanmail.net

${ }^{\dagger}$ The authors contributed equally to this work.
\end{abstract}

Objectives: Despite the low cost and high accessibility of manual acupuncture (MA) treatments for the carpal tunnel syndrome (CTS), this intervention has not been uniformly evaluated in systematic reviews (SR), and no SR has evaluated MA monotherapy for CTS. This review was conducted to summarize the findings and undertake a quality assessment of randomized controlled trials (RCTs) of acupuncture treatment methods for mild-tomoderate CTS to identify clinical evidence for the use of MA in CTS.

Methods: We searched five databases for articles on relevant RCTs that were published until June 2021 without imposing specific restrictions, such as age or sex, on CTS patients. RCTs that evaluated MA were included without any restriction on comparator interventions. Measurement tools for evaluating pain reduction and functional improvement or for evaluating efficacy using electrophysiological indicators were included as outcome measures.

Results: We included seven RCTs, of which three studies reported both post-treatment improvement effects and statistical significance using p-values for all outcomes. Five studies reported statistically significant intergroup differences ( $p$-values for all outcomes) in posttreatment improvement. None of the studies reported severe adverse effects of MA. In all of the RCTs, the reporting rates of the 2c, 2a, and 6a items of STRICTA 2010 were 14\%, $29 \%$, and 29\%, respectively. PC7 (Daereung) was used to treat CTS in all of the included studies.

Conclusion: MA can be used for CTS treatment without serious adverse effects. PC7 was the most commonly used acupoint. In order to ensure objective and reliable reporting, accurate standardization of acupuncture treatment methods acupoint terms should be undertaken in future RCTs.

Keywords: systematic review, carpal tunnel syndrome, manual acupuncture, pain, stricta

\section{INTRODUCTION}

Carpal tunnel syndrome (CTS) is a nerve entrapment syndrome wherein the median nerve is pathologically compressed or damaged, most commonly due to a single neuropathy, within the carpal tunnel [1]. The incidence of CTS increases in populations that habitual perform repetitive hand or wrist motions or use vibrating tools, and CTS has a higher prevalence in women, especially older women, than in men [2]. Risk factors for CTS include diabetes, menopause, hypothyroidism, obesity, arthritis, and pregnancy. CTS is characterized by intermittent, nocturnal paresthesia, and loss of sensation that progressively appears along with weakness and thenar muscle atrophy that results from extensive axonal degeneration [3].

CTS is differentially diagnosed on the basis of clinical assessments and history taking, and definitively diagnosed with electrophysiological diagnostic methods, mainly median nerve conduction velocity (MNCV) [4]. In a study of the cross-sec- 
tional difference between the median nerve within the carpal tunnel and pronator quadratus muscle, CTS patients showed significantly larger cross-sectional differences than controls [5], where differences of more than $2 \mathrm{~mm}^{2}$ were associated with a CTS diagnosis (sensitivity 99\%; specificity 100\%) [5].

For mild-to-moderate CTS, conservative treatment is as effective as conventional treatment [6] for symptom alleviation or functional improvement and includes splints, oral medications, topical steroid injections, electrotherapy, and median nerve slip exercises $[3,7]$. Surgery to relieve pressure in the carpal tunnel is the most effective treatment for severe CTS [8], despite a higher risk of complications, such as hematoma or intra-wound pruritus, than with conservative treatment [6], and caution is needed.

In Korean traditional medicine, CTS belongs to the class of impedimentary diseases, which include paresthesia of muscle, skeleton, and joint; edema; pain; and difficulty in bending and stretching, that are caused by pathogenic Gi of wind, chill, humidity, and fever [9]. In severe cases, these diseases result in an ankylosing joint deformity, which resembles the clinical presentation of CTS [10].

Korean traditional medical treatments for CTS typically include acupuncture, although studies have contradictorily demonstrated the superior and inferior efficacy of acupuncture to placebo or sham acupuncture [11]. Korean studies of acupuncture for CTS include a randomized control trial (RCT) that compared the effectiveness of Sweet Bee Venom pharmacopuncture to that of Scolopendrid pharmacopuncture, case reports of pharmacopuncture (CA; Scolopendrid, CarthmiFlos, and Sweet Bee Venom, along with Jungsongouhyul and Hwangryunhaedok-tang pharmacopunctures) and acupotomy, and papers on acupuncture-based treatment [12, 13]. Systematic reviews (SR) of Korean medical treatments, including acupuncture, for CTS were conducted [11, 14-17]. However, these SR interventions included pharmacopuncture [14], electroacupuncture $[11,16,17]$, laser acupuncture [15, 16], TENS [17], moxibustion [17], TDP lamp [17], and manual acupuncture (MA), and the effectiveness of MA alone has not yet been verified. The recent Korean Medicine Utilization and Herbal Medicine Consumption Survey [18] showed that Korean medical treatment (multiple responses) mainly comprised acupuncture (excluding pharmacopuncture; 93.5\%), followed by moxibustion $(48 \%)$ and pharmacopuncture (22.1\%). The survey indicated that the traditional treatments were considered expensive and the costs included decoctions (75.2\%), pharmacopuncture
(53.1\%), acupuncture (excluding pharmacopuncture; 13.1\%), and moxibustion (11.9\%). Thus, MA is less expensive and more accessible to patients.

This review was conducted to systematically summarize the findings and to conduct a quality assessment of RCTs on acupuncture (MA) treatment methods in mild-to-moderate CTS and to present data that provides clinical evidence for the use of MA in CTS.

\section{MATERIALS AND METHODS}

The protocol of the current systematic review was registered in the PROSPERO International prospective register of systematic reviews (CRD42021258528), and the review was conducted and is reported according to the Preferred Reporting Items for Systematic Reviews and Meta-Analyses (PRISMA) guidelines (2020) [19].

\section{Study subjects and search methods}

\section{1) Criteria for inclusion and exclusion of studies}

In this review, only RCTs aimed at identifying the effectiveness of MA therapy for CTS were include. Therefore, the following literature selection and classification criteria were established according to the Patient population, Intervention, Comparison, Outcomes, and Study design (PICO-SD) guideline.

\section{(1) Study Design}

We selected only RCTs that were reported in Korean or English.

(2) Participants

Studies that enrolled patients diagnosed with mild-tomoderate CTS and treated using electrophysiological diagnostic methods, regardless of their age and sex, were included.

(3) Interventions

Only MA was selected as the intervention, except for hand acupuncture, warm acupuncture, and laser acupuncture, pharmacopuncture, ear acupuncture, electric acupuncture, acupuncture press, etc. There was no restriction on common interventions.

\section{(4) Comparison}

There were no restrictions on the use of comparative interventions.

(5) Outcomes

We selected papers that reported the results of studies that 
used primary measurement tools that objectively evaluated pain reduction and functional improvement, and secondary measurement tools that evaluated treatment effects using electrophysiological indicators.

\section{2) Search strategy}

Four Korean databases [Research Information Sharing Service (RISS), Korean Studies Information Service (KISS), National Digital Science Library (NDSL), and Oriental Medical Advanced Search Integrated System (OASIS)] and one international database (PubMed) were searched for research articles on MA therapy for CTS that were published until June 1, 2021. Detailed searches were conducted with P (Patient) and I (Intervention). Search terms included "Carpal tunnel syndrome," "CTS" and “수근관 증후군” for P and "Acupuncture" and “침" for I. However, in PubMed, we searched for articles using the terms "Carpal Tunnel Syndrome" [Mesh] AND "Acupuncture Therapy" [Mesh], and RCTs were selected through the Article type classification function and were included in the first search.

\section{3) Article screening}

Two researchers (JH and $\mathrm{HI}$ ) independently carried out primary and secondary exclusion of the primarily identified articles based on predefined selection and exclusion criteria. Disagreements, if any, were resolved through a joint review of the paper and, in the absence of consensus, the paper was discussed with a third researcher $(\mathrm{KH})$.

\section{Data extraction}

\section{1) General characteristics of the selected papers}

From the studies that were finally selected, two researchers (JH and HI) independently investigated the authors, publication year, interventions, comparative interventions, sample size, treatment period, outcomes, results, and adverse reactions in the intervention and comparison groups, and disagreements, if any, were resolved through discussion with a third researcher (KH).

\section{2) Evaluation of the quality of acupuncture therapeutic methods based on STRICTA 2010}

The revised Standards for Reporting Interventions in Clinical Trials of Acupuncture (STRICTA 2010) [20] is a formal extension of the Consolidated Standards for Reporting Trials (CONSORT 2010) [21] that is designed to improve the com- pleteness and transparency of intervention reporting in controlled clinical trials of acupuncture, as described in Item 5 (intervention) of CONSORT 2010. Based on the STRICTA 2010 guidelines, in this study, acupuncture methods were independently investigated by two researchers ( $\mathrm{JH}$ and $\mathrm{HI}$ ) and, in the event of disagreement, were discussed with a third researcher $(\mathrm{KH})$.

\section{Methodological quality assessment of the included studies}

\section{1) Risk of bias}

Two researchers (JH and $\mathrm{HI}$ ) evaluated the selected papers for Risk of Bias (ROB) using the evaluation list provided by the Cochrane Handbook for Systematic Reviews of Interventions [22]. ROB was evaluated in six areas (sequence generation, allocation sequence concealment, blinding of participants and personnel, blinding of outcome assessment, incomplete outcome data, and selective outcome reporting) for each selected paper, and each item was graded as low, uncertain, or high risk by two independent researchers ( $\mathrm{JH}$ and $\mathrm{HI}$ ), with disagreements resolved by discussion with a third researcher $(\mathrm{KH})$.

\section{RESULTS}

\section{Results of the literature search}

In the first search of five databases, a total of 281 papers were retrieved (RISS: 77; KISS: 30; NDSL: 140; OASIS: 17; and PubMed: 17) and titles were extracted. Among these, $170 \mathrm{du}-$ plicate papers were automatically excluded using MS Excel or by manually checking authors' names, date of publication, and the full-text article. On screening the titles and abstracts, we excluded 31 case reports, 20 non-original papers (e.g., review articles, SR, and protocol studies), and 23 non-CTS studies. After the abovementioned initial exclusion, we classified the remaining papers using the Study Design Algorithm for Medical Literature of Intervention (DAMI) [23] of the full-text articles, and excluded non-RCT papers and papers whose original texts could not be accessed; thus, 1 article on a case-control study, 5 on functional magnetic response imaging (fMRI), 16 whose original texts could not be accessed, 6 RCTs using interventions other than MA, and 1 prospective observational study were secondarily excluded. Seven papers were selected for inclusion in the analysis (Fig. 1). 


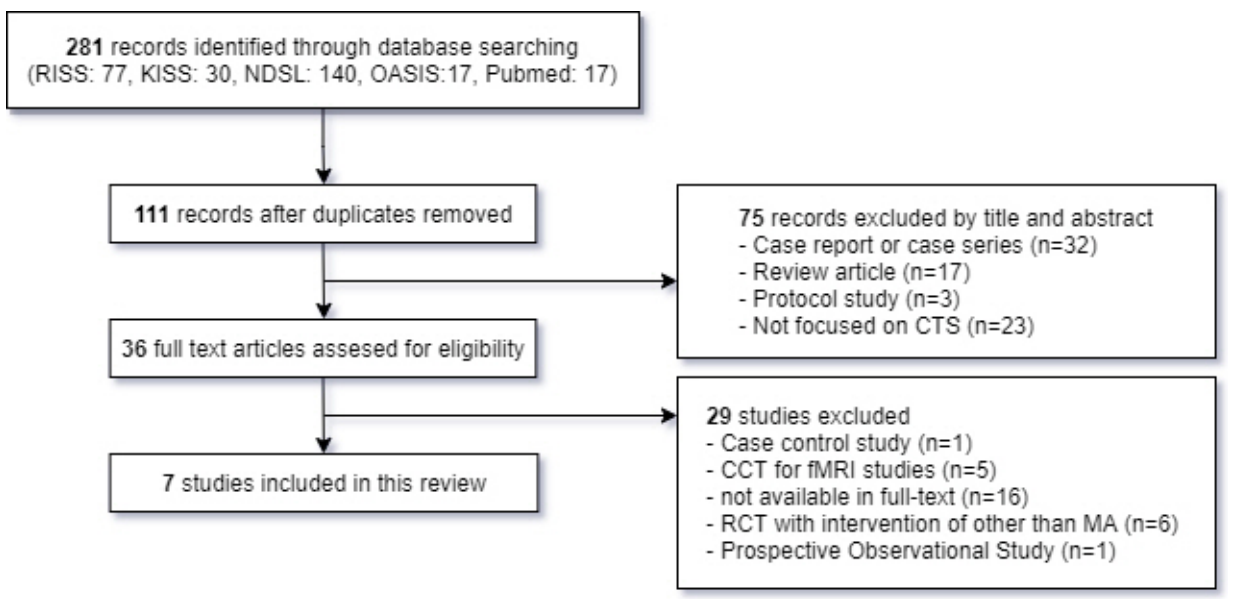

Figure 1. Flow diagram for selection of studies.

\section{General characteristics of selected papers}

\section{1) Treatment methods (interventions, comparative interventions, and common intervention) and study period}

In all seven of the selected papers [24-30], MA was chosen as the intervention. For comparison, we included two studies $[25,26]$ without treatment, two with NSAIDs (Celebrex [24] and ibuprofen [27]), one with prednisolone [30], and two with sham acupuncture (SA) $[28,29]$. As a common intervention, night segments were used in all but one study [30]. The treatment periods were similar for the common intervention and for the intervention, and most frequently was 1 month (4 weeks) in five papaers $[24,26,27,29,30]$, although there was one paper each of 5 [25] and 6 weeks [28] duration (Table 1).

\section{2) Measurement tools and results of MA}

The Global Symptom Score (GSS) for symptoms [24, 29, 30]; Carpal Tunnel Self-Assessment Questionnaire (CTSAQ) [28]; Boston Carpal Tunnel Questionnaire (BCTQ) for symptom and function [25, 27]; Quick Disabilities of the Arm, Shoulder, and Hand (Quick DASH) [26]; Duruoz Hand Index (DHI) for function and disability [26]; visual analogue scale (VAS) for pain [25-27], and Nottingham Health Profile (NHP) for quality of life [25] were used as the main measurement tools.

With regard to electrophysiological assessments methods, the distance motor latency (DML) [24-26, 29, 30], distal sensory latency (DSL) $[24,27,29,30]$, compound muscle action potential (CMAP) [24, 26, 30], sensory nerve action potential (SNAP) $[24,26,30]$, nerve conduction velocity (NCV) [24-27, $29,30]$, in many ways, and motor amplitude [25] were used as secondary measurement tools. Additionally, the median nerve cross-sectional area (CSA) [26] (measured using ultrasound), key and tip pinch assessment [28], and the combined sensory index (CSI) [28] were ascertained.

In the same group, three papers $[26,27,29]$ reported both the results of post-treatment improvement and statistical significance (using p-values); three papers $[24,28,30]$ did not report p-values with statistical significance, and one paper [25] did not report all results. Five papers [24-27, 29] reported both the results and statistically significant differences in post-treatment improvement effects between the intervention and control groups, one paper [30] did not report p-values when there was no statistical significance, and one paper [28] did not report some results (Table 1).

\section{3) Adverse effects}

Four of the seven papers $[25,27,28,30]$ reported posttreatment non-serious adverse effects, including erythema [25] as well as local pain, ecchymosis, and local paresthesia [30]. In comparison, ibuprofen caused gastrointestinal side effects [27], which were treated with omeprazole, whereas prednisolone caused nausea and epigastralgia [30] in 18\% of the control group, which resulted in four patients dropping out (Table 1).

\section{Analyzing the quality of reporting acupuncture treatment methods using STRICTA 2010}

The itemized reporting rates for STRICTA 2010 for the seven selected papers were $100 \%$ in 2b, 2e, 2f, $2 \mathrm{~g}, 3 \mathrm{a}, 3 \mathrm{~b}, 4 \mathrm{a}, 4 \mathrm{~b}$, and $5 ; 71 \%$ for $1 \mathrm{a}$ and $1 \mathrm{~b} ; 43 \%$ for $2 \mathrm{~d} ; 29 \%$ for $2 \mathrm{a}$; and $14 \%$ for 2c. 


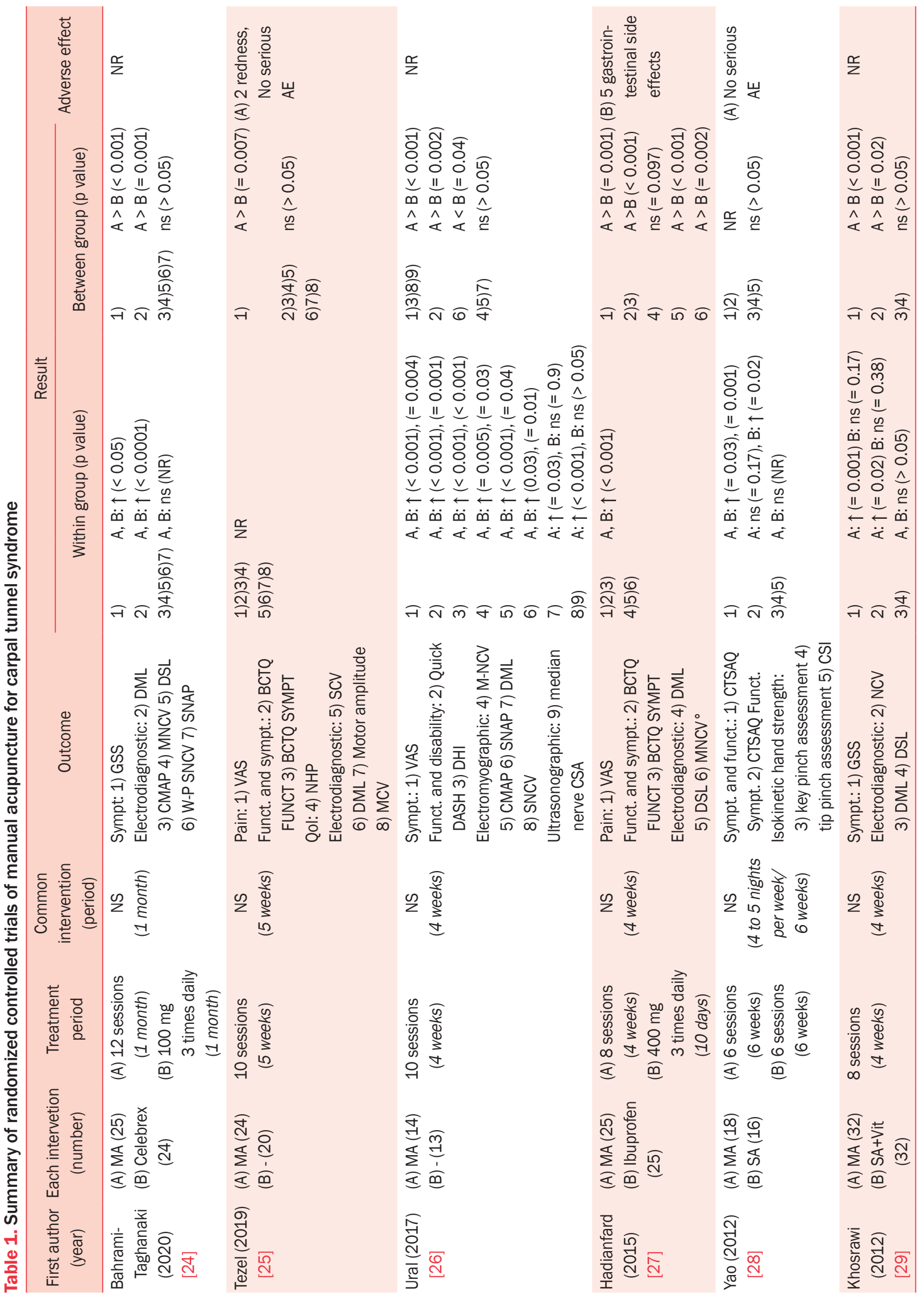




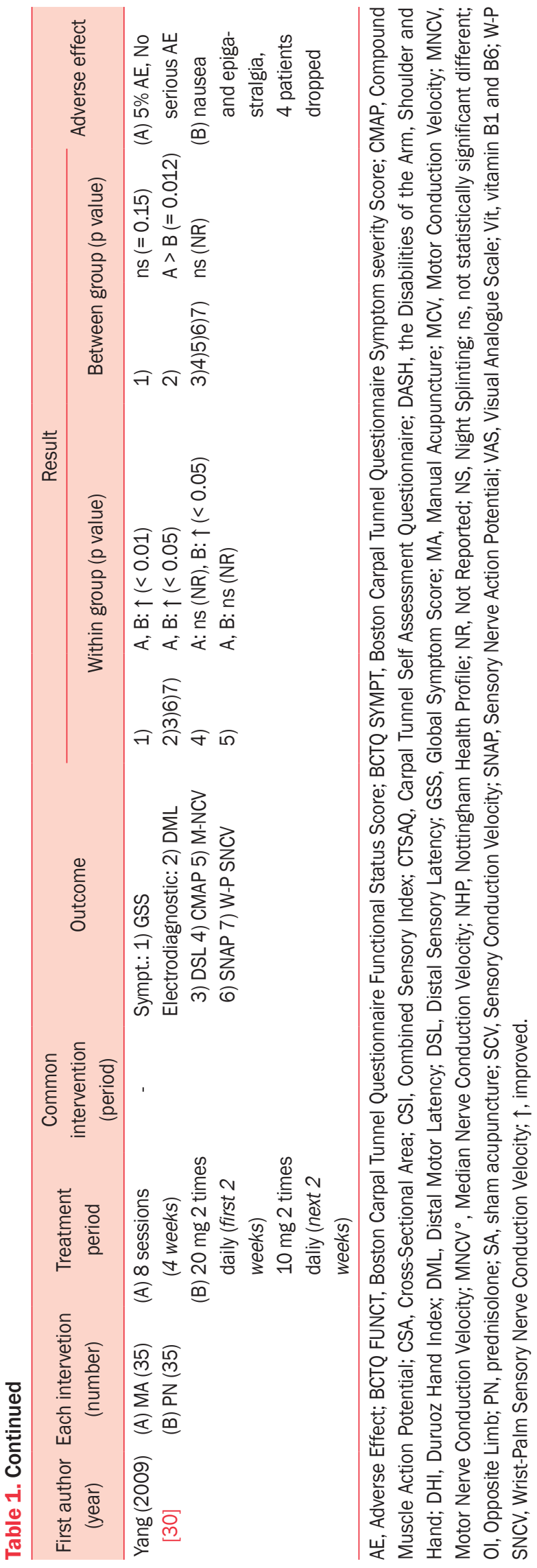

\section{1) Acupuncture rationale}

With regard to the style of acupuncture, three papers researched Comprehensive and Alternative Medicine (CAM; $42.8 \%$ ), two evaluated Traditional Chinese Medicine (TCM; $28.6 \%$ ), and two did not report the style (28.6\%). In terms of the treatment rationale, two papers were identified as previous studies (28.6\%), two did not report (28.6\%), one was identified as Laws of TCM (14.3\%), Acupuncture literature [31] (14.3\%), and one was identified as TCM Textbooks \& Acupuncturists consensus (14.3\%). Among them, the paper that reported "Acupuncture literature" only had references. An evaluation of the extent of treatment variation showed that three papers reported consistent treatment $(42.8 \%)$, three mentioned treatment changes depending on the patient's condition (42.8\%), and one did not report (14.3\%) (Table 2).

\section{2) Details of needling}

In terms of the number of needle insertions, five papers did not report the number of needle insertions per subject as a simple total (71.4\%), one reported nine insertions (14.3\%), and one reported six insertions (14.3\%). All papers reported the names of the acupoints used. PC7 was most frequently used among the 43 acupoints in all seven papers. Sequentially, PC6 was used in six papers; LI11 in five papers; HT7, LU9, PC4, and PC8 were used in three studies; and HT2, HT8, and LI4 were used in two studies. GB34, SI3, ST36, SP6, TB4, TB5, and TH5 were used in one study. Of the two studies that used SA as a comparison intervention, one study used the same acupoints as the intervention group, and one study used the same acupoints as the intervention group. Six papers did not report the depth of insertion (85.7\%); one reported 0.5 to 1.0 inches at PC7 and 1.0 to 1.5 inches at PC6 (14.3\%), and the depth of insertion varied by location. Four papers reported the form of acupuncture stimuli simply as MA (57.2\%), and three (42.8\%) reported a special sensation called “de qi (得氣)": two wherein the needles were manipulated by twirling with lifting-thrusting methods for de $q i$, and one that did not report any needle-stimulation techniques. For bedtime, three, two, one, and one papers set $20(42.8 \%), 30(28.6 \%), 25(14.3 \%)$, and 60 (14.3\%) minutes, respectively. All seven papers (100\%) reported the use of 0.25 mm-diameter needles (Table 2).

\section{3) Treatment regimen}

With regard to the number of treatment sessions, three, two, and one papers reported 8 (42.8\%), 10 (28.6\%), 12 (14.3\%), and 


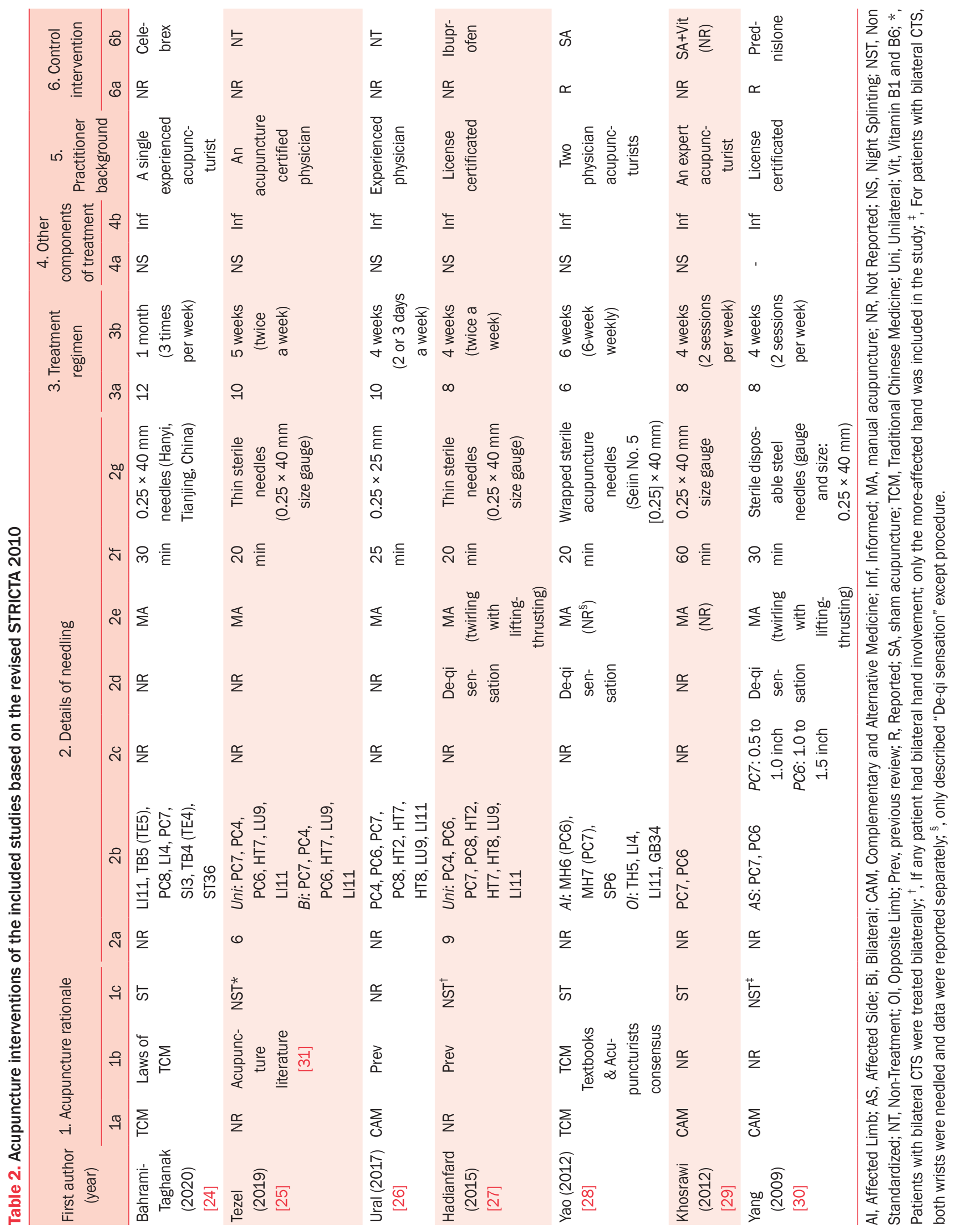


6 (14.3\%) sessions, respectively. Treatment frequency was documented as regular weekly treatments in six papers and as a total of 10 treatments, with two or three treatments per week, in one paper. Of the six papers with a regular weekly treatments, four papers reported two treatment sessions per week, one paper had three treatments per week, and one paper had three treatments per week (Table 2).

\section{4) Other components of treatment}

The other interventions administered to the acupuncture group included night splints (in six papers, 85.7\%); one paper did not report any treatment except acupuncture (14.3\%). All seven papers $(100 \%)$ presented information on the treatment environment and practitioners (Table 2).

\section{5) Practitioner background}

The practitioners included acupuncturists (three papers; $42.8 \%$ ), licensed practitioners (two papers; $28.6 \%$ ), and physicians (two papers; $28.6 \%$ ) (Table 2).

\section{6) Control or comparative interventions}

Five papers $(71.4 \%)$ did not report a rationale for the comparator intervention in the context of the research question, with sources that justify this choice, whereas two papers (28.6\%) reported this aspect. Six papers $(85.7 \%)$ provided a precise description of the comparator intervention, whereas one $(14.3 \%)$ did not (Table 2).

\section{Risk of bias assessment}

The risk of bias in the seven papers was assessed by applying the Cochrane Risk of Bias criteria (Fig. 2).

\section{1) Random sequence generation}

All seven papers were judged as showing low risk of random allocation order generation.

\section{2) Allocation concealment}

All seven papers were judged to show unclear risk of allocation concealment because they did not report the method of allocation concealment.

\section{3) Blinding of participants and personnel}

All seven papers were judged to show a high risk of bias of blinding of participants and personnel due to the nature of

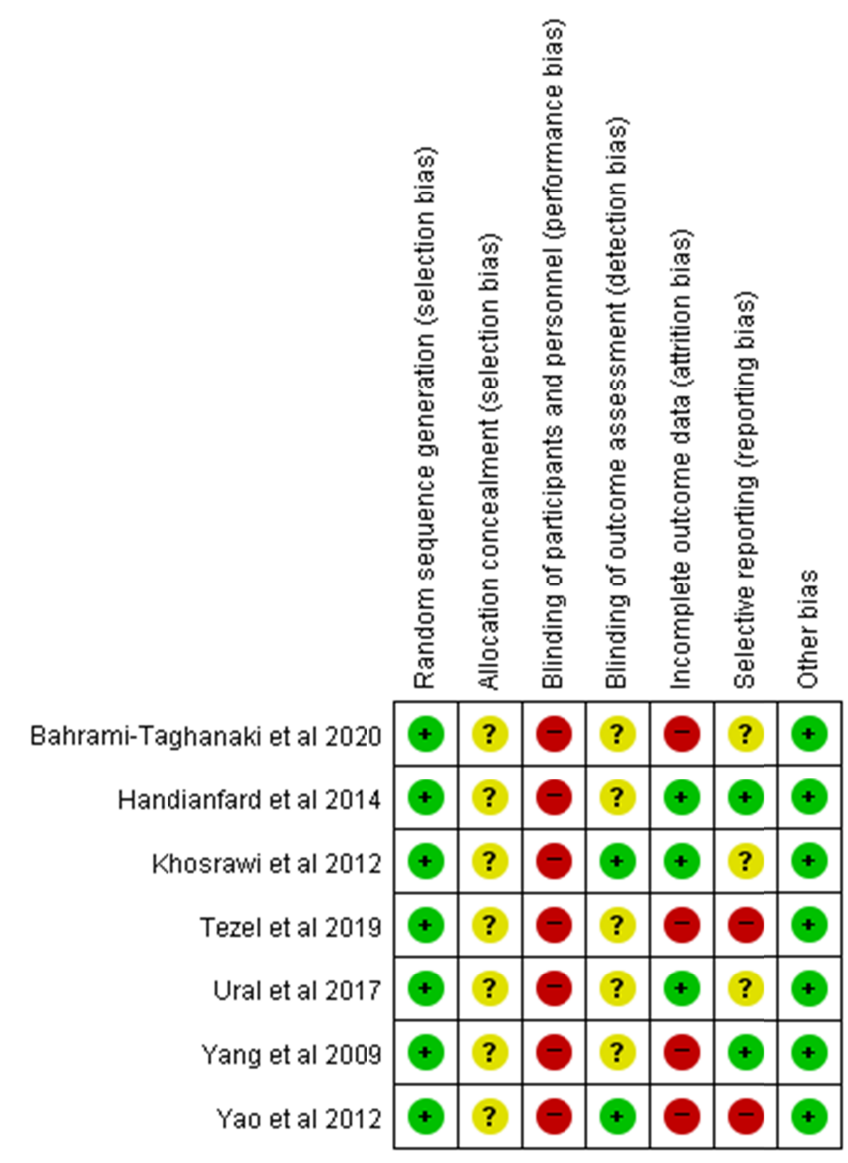

Figure 2. Risk of bias assessed using the Cochrane "Risk of bias" tool. +, low risk of bias; -, high risk of bias; ?, unclear risk of bias.

RCTs on acupuncture.

4) Blinding of outcome assessment

Five papers [24-27, 30] were judged to show an unclear risk of bias as the method of blinding of outcome assessment was not reported. The other five papers $[28,29]$ were judged as showing a low risk of bias.

\section{5) Incomplete outcome data}

Four papers [24, 25, 28-30] were judged to show a high risk of bias, as missing data seemed to have affected the outcomes. The other three papers $[28,29]$ were judged to show a low risk of bias.

\section{6) Selective reporting}

Three papers $[24,26,29]$ were judged to show an unclear risk of bias, because they did not report adverse effects. Moreover, two papers $[25,28]$ were judged to show a high risk of bias as some of the results were not reported. The remaining two 
papers were judged to have a low risk of bias.

\section{7) Other bias}

All seven papers were judged to show a low risk of other bias.

\section{DISCUSSION}

CTS is a neuropathy caused by compression of the median nerve within the carpal tunnel [32]. Treatment-related decisions are determined based on the severity of clinical symptoms of CTS [33], and the measurement of the MNCV is the main method for definitive diagnosis of CTS [4]. However, because MNCV does not provide information on pathological and anatomical abnormalities in surrounding tissues, ultrasound has recently been used to diagnose CTS based on differences in the cross-sectional area of the median nerve located in the carpal tunnel and the rectangular gyrus muscle [3, 33]. Furthermore, studies have shown that ultrasound is helpful for diagnosing CTS, despite the inability to assess the severity of CTS [34].

In Korea, SRs have evaluated Korean medical treatments, including acupuncture, for CTS [11, 14-17]. However, these SRs included interventions such as pharmacopuncture [14], electroacupuncture $[11,16,17]$, laser acupuncture $[15,16]$, TENS [17], moxibustion [17], and TDP lamp [17] with MA. However, the effectiveness of MA as monotherapy has not been verified yet. Therefore, in this study, we summarized the findings and undertook a quality assessment of reports of acupuncture treatment methods from RCTs of MA for mild-to-moderate CTS. In all seven papers, MA was the intervention and the comparator interventions included NSAIDs in three and SA in two studies. As a common intervention, night splints were used in all but one study. De Angelis et al. (2009) [35] reported that night splint treatment is effective for improving symptoms and function in patients with CTS.

With regard to the main measurement tools, GSS for symptoms and VAS for pain were used most frequently, followed by the BCTQ and CTSAQ, which simultaneously evaluate symptoms and functional improvement. Among the secondary measurement tools, different methods to evaluate NCV were used most often, followed by DML, DSL, and SNAP.

No significant adverse effect of MA was reported in any study. However, adverse effects of the comparator intervention included gastrointestinal side effects due to ibuprofen as well as nausea and gastric pain due to prednisolone. In particular, the adverse effects of prednisolone decreased the number of participants in clinical trials. The abovementioned findings confirm the safety of MA, which has no serious side effects.

This study used STRICTA 2010 checklist to evaluate the quality of reporting of MA intervention methods in the selected RCTs. To prevent errors in translation, we referred to the STRICTA 2010 checklist that was previously translated into Korean [36]. The reporting rates for 17 items of STRICTA 2010 for the seven papers showed that none of the papers reported all the items, and the paper that reported the most items $(n=15$; 88.2\%) was by Yang et al. (2009) [30]. The paper that reported the least number of items $(n=11 ; 64.7 \%)$ was by Khosrawi et al. (2012) [29]. On average, the seven selected papers reported 13.1 items (77\%). The paper by Yang et al. (2009) [30], which was published before the STRICTA guideline was revised in 2010, had the highest reporting rate. However, no paper had a $100 \%$ reporting rate, suggesting that future RCTs need to elucidate the reporting of MA intervention. Furthermore, there 2c (14\%), 2a (29\%), and $6 \mathrm{a}(29 \%)$ items had a reporting rate of less than $30 \%$. Therefore, we suggest a need for future RCTs to describe the MA intervention by reporting the depth of insertion (including units of measure) (2c), number of needle insertions per subject per session (2a), and the rationale for the control or comparator in the context of the research question, with sources that justify this choice (6a).

PC7 was used in all of the seven papers, whereas PC6; LI11; HT7, LU9, PC4, and PC8; HT2, HT8, and LI4; and GB34, SI3, ST36, SP6, TE4, TE5, and TH5 were used in six, five, three, two, and one paper(s), respectively. The acupoints used in more than three papers were all located below the elbow joint, and most of the practitioners chose points around the wrist as treatment sites.

Yao et al. (2012) [28] reported MH7 and MH6, instead of PC7 and PC6, which are the WHO standard acupoints. Bahrami-Taghanak et al. (2020) [24] reported TB4 and TB5, instead of TE4 and TE5, which are the WHO standard acupoints. Nonetheles, there is no confusion when the exact name of the acupoints is indicated, and the reproducibility of the treatment effects could be increased; therefore, exact labeling of the acupoints is required.

In the most recent SR on the reporting quality of acupuncture treatment for CTS, Hyun et al. (2020) [11] stated that the paper by Ural and Öztürk (2017) [26] reported the use of nine needles per patient in the $2 \mathrm{a}$ item although the study did not report the total number of needle insertions per subject (2a). 
Therefore, in this study, we report that this item as unreported in that paper. Moreover, Hyun et al. (2020) stated that the paper by Ural and Öztürk (2017) [26] reported a "previous study" as the rationale for the control or comparator in the context of the research question, with sources that justified this choice (6a). However, we reported this item as "not reported." Furthermore, those researchers reported that the paper by Yao et al. (2012) [28] reported "MA" for the needle stimulation (2e). Although the paper reported that the practitioner caused a de qi sensation, it did not report the techniques for inducing the de qi sensation; therefore, we reported this item as "not reported." In this study, we determined that the explanation of the STRICTA 2010 items should have been satisfied.

For the assessment of the risk of bias, the seven papers included in this analysis were mostly rated as having a "low" or "unclear" risk of bias. The most uncertain risk of ROB item was the "Blinding of participants and personnel," as this constitutes an inherent limitation of acupuncture research.

In the selected papers, several measurement tools measured the post-treatment improvement of patients and, in most cases, a comparison of the MA and control groups showed that MA treatment was significantly more effective than the comparator intervention. Thus, MA treatment was positively evaluated with regard to efficacy for mild-to-moderate CTS.

The main limitation of this study was that all of the selected RCTs were reported in international papers, making it difficult to identify the trends in Korean RCTs. Furthermore, there were four identical papers among the selected RCTs that were comparable to the existing SR by Hyun et al. (2020) [11]. Nonetheless, this review is meaningful because we evaluated the recent RCTs reported in 2020 [24] and 2019 [25], the effectiveness of MA on CTS, and the quality of MA treatment methods, and the results of this SR can potentially be used as basic data for generating clinical evidence for MA treatment of CTS.

\section{CONCLUSIONS}

Based on STRICTA 2010 guideline, we conducted a comprehensive literature review of RCTs that investigated MA treatment for mild-to-moderate CTS and reached the following conclusions:

1. With regard to adverse effects, MA treatment, unlike conventional medicine which has serious side effects, showed no serious side effects in papers that reported adverse effects.

2. PC7 was the most commonly used acupoint, followed by
PC6 and LI11. Most of the remaining acupoints were located below the elbow joint.

3. To minimize confusion, we recommend that future RCTs use the WHO standard acupoints when reporting acupoints.

4. Among the items of the STRICTA 2010 guideline, the depth of insertion (including units of measure; $2 \mathrm{c}$ ), number of needle insertions per subject per session (2a), and rationale for the control or comparator in the context of the research question, with sources that justify this choice (6a) had lower reporting rates; therefore, we suggest that future RCTs should accurate report these items.

\section{ACKNOWLEDGMENTS}

This study was edited to ensure language and grammar accuracy by Editage, a division of Cactus Communications.

\section{AUTHORS' CONTRIBUTIONS}

Conceptualization: JH. Methodology: JH. Software: JH. Validation: JH. Formal analysis: JH, HI. Investigation: JH, HI. Resources: HI. Data curation: JH, HI. Writing - Original Draft: JH. Writing - Review \& Editing: JH, HI, KH. Visualization: JH, HI. Supervision: KH. Project administration: KH. Funding acquisition: $\mathrm{KH}$.

\section{CONFLICT OF INTEREST}

The authors declare that they have no conflicts of interest.

\section{FUNDING}

This study was supported by grants from the National Research Foundation of Korea (NRF-2019R1C1C1007573) funded by the Korean government.

\section{ETHICAL STATEMENT}

No ethical approval was required as this study did not involve human participants or laboratory animals.

\section{DATA AVAILABILITY}

The authors state that all information provided in this article can be obtained from the author on request. 


\section{ORCID}

Jeong Ho Huh, https://orcid.org/0000-0002-8283-6340

Hye In Jeong, https://orcid.org/0000-0002-3651-9678

Kyeong Han Kim, https://orcid.org/0000-0003-4868-9145

\section{REFERENCES}

1. Urits I, Gress K, Charipova K, Orhurhu V, Kaye AD, Viswanath O. Recent advances in the understanding and management of carpal tunnel syndrome: a comprehensive review. Curr Pain Headache Rep. 2019;23(10):70.

2. Atroshi I, Gummesson C, Johnsson R, Ornstein E, Ranstam J, Rosén I. Prevalence of carpal tunnel syndrome in a general population. JAMA. 1999;282(2):153-8.

3. Padua L, Coraci D, Erra C, Pazzaglia C, Paolasso I, Loreti C, et al. Carpal tunnel syndrome: clinical features, diagnosis, and management. Lancet Neurol. 2016;15(12):1273-84.

4. Dabbagh A, MacDermid JC, Yong J, Macedo LG, Packham TL. Diagnosing carpal tunnel syndrome: diagnostic test accuracy of scales, questionnaires, and hand symptom diagrams-a systematic review. J Orthop Sports Phys Ther. 2020;50(11):622-31.

5. Klauser AS, Halpern EJ, De Zordo T, Feuchtner GM, Arora R, Gruber J, et al. Carpal tunnel syndrome assessment with US: value of additional cross-sectional area measurements of the median nerve in patients versus healthy volunteers. Radiology. 2009;250(1):171-7.

6. Shi Q, MacDermid JC. Is surgical intervention more effective than non-surgical treatment for carpal tunnel syndrome? A systematic review. J Orthop Surg Res. 2011;6:17.

7. Jiménez Del Barrio S, Bueno Gracia E, Hidalgo García C, Estébanez de Miguel E, Tricás Moreno JM, Rodríguez Marco S, et al. Conservative treatment in patients with mild to moderate carpal tunnel syndrome: a systematic review. Neurologia (Engl Ed). 2018;33(9):590-601.

8. Pourmokhtari M, Mazrooyi M, Vosoughi AR. Conservative or surgical treatment of carpal tunnel syndrome based on the severity and patient risk factors. Musculoskelet Surg. 2021;105(3): 315-9.

9. Lee JH. Korean medicine for carpal tunnel syndrome: a systematic review of literature published in Korea. J Physiol Pathol Korean Med. 2015;29(1):79-84.

10. Kim ID, Oh HH, Byun JY, Moon HC, Koh KH, Park SY, et al. Clinical study on the patients with carpal tunnel syndrome. J Korean Acupunct Moxib Soc. 2002;19(6):61-6.

11. Hyun JY, Shin J, Im CJ, Park J. A systematic review on the reporting quality of acupuncture treatment for carpal tunnel syn- drome. Korean J Acupunct. 2020;37(3):131-44.

12. Jeon HA, Shin WB, Lim SY, Moon YJ, Lee JY, Ku SH, et al. A narrative review of carpal tunnel syndrome in traditional Chinese medicine: using China National Knowledge Infrastructure database. J Korean Med Rehabil. 2020;30(4):119-32.

13. Lim NR, Kim SC, Jang EH, Na WM, Lim SI, Shin JB, et al. Case study of oriental medicine treatment with acupotomy therapy of the carpal tunnel syndrome. J Korean Acupunct Moxib Soc. 2008;25(4):163-70.

14. Kim JH, Song HS. Traditional Korean medicine therapy for treating carpal tunnel syndrome in patients with wrist pain: a systematic review. Acupuncture. 2015;32(2):59-64.

15. Sim H, Shin BC, Lee MS, Jung A, Lee H, Ernst E. Acupuncture for carpal tunnel syndrome: a systematic review of randomized controlled trials. J Pain. 2011;12(3):307-14.

16. Choi GH, Wieland LS, Lee H, Sim H, Lee MS, Shin BC. Acupuncture and related interventions for the treatment of symptoms associated with carpal tunnel syndrome. Cochrane Database Syst Rev. 2018;12(12):CD011215.

17. Wu IX, Lam VC, Ho RS, Cheung WK, Sit RW, Chou LW, et al. Acupuncture and related interventions for carpal tunnel syndrome: systematic review. Clin Rehabil. 2020;34(1):34-44.

18. Ministry of Health and Welfare. Korean Medicine Utilization and Herbal Medicine Consumption Survey (KOSIS No. 117087) [Internet]. Sejong: Ministry of Health and Welfare; 2018 [cited 2021 Jun 10]. Available from: http://www.mohw. go.kr/react/jb/sjb030301vw.jsp?PAR_MENU_ID=03\&MENU_ ID $=032901 \&$ CONT_SEQ=344055.

19. Page MJ, McKenzie JE, Bossuyt PM, Boutron I, Hoffmann TC, Mulrow CD, et al. The PRISMA 2020 statement: an updated guideline for reporting systematic reviews. BMJ. 2021;372:n71.

20. MacPherson H, Altman DG, Hammerschlag R, Li Y, Wu T, White A, et al. Revised STandards for Reporting Interventions in Clinical Trials of Acupuncture (STRICTA): extending the CONSORT statement. Acupunct Med. 2010;28(2):83-93.

21. Moher D, Hopewell S, Schulz KF, Montori V, Gøtzsche PC, Devereaux PJ, et al. CONSORT 2010 explanation and elaboration: updated guidelines for reporting parallel group randomised trials. Int J Surg. 2012;10(1):28-55

22. Higgins JPT, Thomas J, Chandler J, Cumpston M, Li T, Page MJ, et al. Cochrane handbook for systematic reviews of interventions. 2nd ed. Chichester: Wiley; 2019.

23. Seo HJ, Kim SY, Lee YJ, Jang BH, Park JE, Sheen SS, et al. A newly developed tool for classifying study designs in systematic reviews of interventions and exposures showed substantial reliability and validity. J Clin Epidemiol. 2016;70:200-5.

24. Bahrami-Taghanaki $\mathrm{H}$, Azizi $\mathrm{H}$, Hasanabadi $\mathrm{H}$, Jokar $\mathrm{MH}$, Iranmanesh A, Khorsand-Vakilzadeh A, et al. Acupuncture for 
carpal tunnel syndrome: a randomized controlled trial studying changes in clinical symptoms and electrodiagnostic tests. Altern Ther Health Med. 2020;26:10-6.

25. Tezel N, Umay E, Yilmaz V, Cakci A. Acupuncture plus night splint for quality of life and disability in patients with carpal tunnel syndrome: a randomized controlled trial. Integr Med Res. 2019;8(4):284-8.

26. Ural FG, Öztürk GT. The acupuncture effect on median nerve morphology in patients with carpal tunnel syndrome: an ultrasonographic study. Evid Based Complement Alternat Med. 2017;2017:7420648.

27. Hadianfard M, Bazrafshan E, Momeninejad H, Jahani N. Efficacies of acupuncture and anti-inflammatory treatment for carpal tunnel syndrome. J Acupunct Meridian Stud. 2015;8(5):229-35.

28. Yao E, Gerritz PK, Henricson E, Abresch T, Kim J, Han J, et al. Randomized controlled trial comparing acupuncture with placebo acupuncture for the treatment of carpal tunnel syndrome. PM R. 2012;4(5):367-73.

29. Khosrawi S, Moghtaderi A, Haghighat S. Acupuncture in treatment of carpal tunnel syndrome: a randomized controlled trial study. J Res Med Sci. 2012;17(1):1-7.
30. Yang CP, Hsieh CL, Wang NH, Li TC, Hwang KL, Yu SC, et al. Acupuncture in patients with carpal tunnel syndrome: a randomized controlled trial. Clin J Pain. 2009;25(4):327-33.

31. Freedman J. Acupuncture for carpal tunnel syndrome. Acupunct Med. 2002;20(1):39-40.

32. Ashworth NL. Carpal tunnel syndrome. BMJ Clin Evid. 2010; 2010:1114

33. Jung IY, Kim NH, Jeong KS, Yang H, Park KS. Assessment of severity by high resolution ultrasonography in carpal tunnel syndrome. J Korean Neurol Assoc. 2012;30(3):176-81.

34. Ozsoy-Unubol T, Bahar-Ozdemir Y, Yagci I. Diagnosis and grading of carpal tunnel syndrome with quantitative ultrasound: is it possible? J Clin Neurosci. 2020;75:25-9.

35. De Angelis MV, Pierfelice F, Di Giovanni P, Staniscia T, Uncini A. Efficacy of a soft hand brace and a wrist splint for carpal tunnel syndrome: a randomized controlled study. Acta Neurol Scand. 2009;119(1):68-74.

36. Lee HS, Cha SJ, Park HJ, Seo JC, Park JB, Lee HJ. Revised STandards for Reporting Interventions in Clinical Trials of Acupuncture (STRICTA): extending the CONSORT statement. Korean J Acupunct. 2010;27(3):1-23. 